\title{
Simple API for XML
}

National Cancer Institute

\section{Source}

National Cancer Institute. Simple API for XML. NCI Thesaurus. Code C73931.

A computer program that divides code up into functional components. 